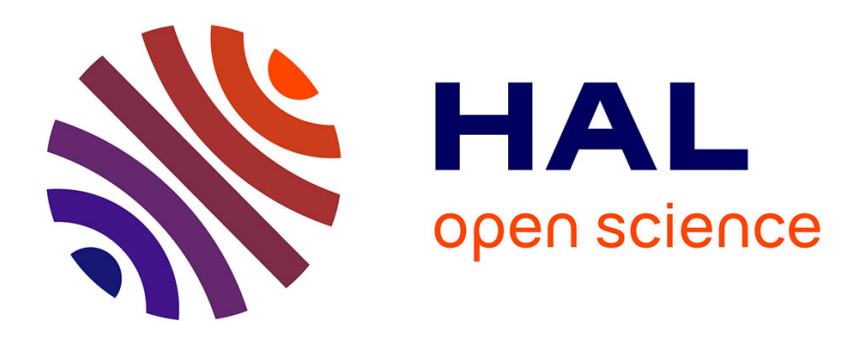

\title{
Engineering glowing chemogenetic hybrids for spying on cells
}

\author{
Hela Ben Aissa, Arnaud Gautier
}

\section{To cite this version:}

Hela Ben Aissa, Arnaud Gautier. Engineering glowing chemogenetic hybrids for spying on cells. European Journal of Organic Chemistry, 2020, 10.1002/ejoc.202000340 . hal-03033405

\section{HAL Id: hal-03033405 \\ https://hal.sorbonne-universite.fr/hal-03033405}

Submitted on 1 Dec 2020

HAL is a multi-disciplinary open access archive for the deposit and dissemination of scientific research documents, whether they are published or not. The documents may come from teaching and research institutions in France or abroad, or from public or private research centers.
L'archive ouverte pluridisciplinaire HAL, est destinée au dépôt et à la diffusion de documents scientifiques de niveau recherche, publiés ou non, émanant des établissements d'enseignement et de recherche français ou étrangers, des laboratoires publics ou privés. 


\title{
Engineering glowing chemogenetic hybrids for spying on cells
}

\author{
Hela Ben Aissa, ${ }^{[a]}$ and Arnaud Gautier ${ }^{*[a, b]}$
}

[a] H. Ben Aissa, Prof. Dr. A. Gautier

Sorbonne Université, École normale supérieure, PSL University, CNRS, Laboratoire des biomolécules, LBM, 75005 Paris, France

arnaud.gautier@sorbonne-universite.fr

[b] Prof. Dr. A. Gautier

Institut Universitaire de France

\begin{abstract}
Seeing biochemical events is essential to understand the functioning of cells and organisms. Such imaging depends on the selective targeting of fluorescent reporters and biosensors in living systems. These reporters and biosensors can be entirely synthetic, entirely genetically encoded or hybrid. Hybrid reporters and biosensors composed of a genetically encoded module that binds a fluorogenic chromophore and activates its fluorescence have recently drawn attention as they offer new opportunities for pushing the frontiers of bioimaging.
\end{abstract}

(GFP) discovered in Aequorea Victoria jellyfish and the red fluorescent protein (RFP) found in Discosoma sp. mushroom anemone. Fluorescent proteins are structurally homologous and share the unique property of forming in presence of molecular oxygen a fluorescent chromophore from three amino acid residues within their own polypeptide sequence. Thanks to extensive engineering efforts, a broad palette of fluorescent proteins with various colors and improved photophysical and photochemical properties is now available ${ }^{[2]}$. The concomitant development of directed evolution techniques has been essential for engineering fluorescent proteins that are more photostable and monomeric [3] [4]

The fluorescence toolbox has been recently expanded with the discovery and development of alternative chemogenetic hybrid fluorescent labels. These systems open new prospects for biological imaging as they circumvent some shortcomings of fluorescent proteins - such as their large size (25-30 kDa), their slow fluorescence maturation and their need for molecular oxygen to fluoresce ${ }^{[5]}$. Among these systems, some come from Nature such as the small oxygen-insensitive green fluorescent protein UnaG, found in Japanese eel, which fluoresces thanks to the binding of bilirubin (BR), a catabolic product of heme present in cells $^{[6]}$. But most of them have been developed through engineering. They are in general composed of a genetically encoded tag designed to form a fluorescent complex with a small chromophore (Figure 1). These chromophores are eventually fluorogenic: essentially non-fluorescent when free in solution, they strongly fluoresce when bound to their cognate tag. Fluorogenic chromophores are preferred as they provide higher contrast for imaging than classical fluorophores. By engineering the tag through e.g. rational design, directed evolution or de novo design, and/or the fluorogenic chromophores (also called fluorogens) by molecular engineering, chemists and biologists have built and breed a large collection of hybrid chemogenetic reporters allowing the efficient imaging of proteins - but also of other biomolecules such as RNA - and the creation of actuators and biosensors for various applications in Biology.

\section{Hybrid reporters}

Biological imaging has become an essential tool in Biology. Fluorescent reporters enable to see biochemical events inside living cells and organisms with high spatiotemporal resolution. The revolution came with the development of genetically encoded reporters, which enables to easily and specifically insert the instructions for their synthesis into cells in the form of DNA. Fused to a protein of interest (or expressed in a particular cell type), genetically encoded fluorescent reporters enable selective labeling, tracking and localization of proteins (or cells) in living systems ${ }^{[1]}$. The best-known example of such reporters are fluorescent proteins derived from the green fluorescent protein

\subsection{Tag engineering}

The design of fluorescent chemogenetic hybrid reporters has largely benefited from the development of protein and nucleic acid engineering. Programming new functions in proteins or nucleic acids can be done nowadays using various approaches based either on rational design, directed evolution or de novo design. Rational design can be efficient in reprogramming the function of an existing protein using structural information and site-directed mutagenesis. Directed evolution is useful for improving properties or programing new functions in a given protein or nucleic acid 


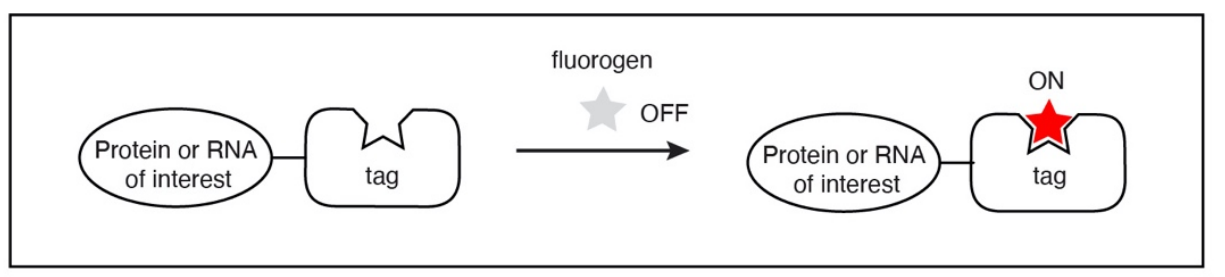

tag engineering

fluorogen engineering
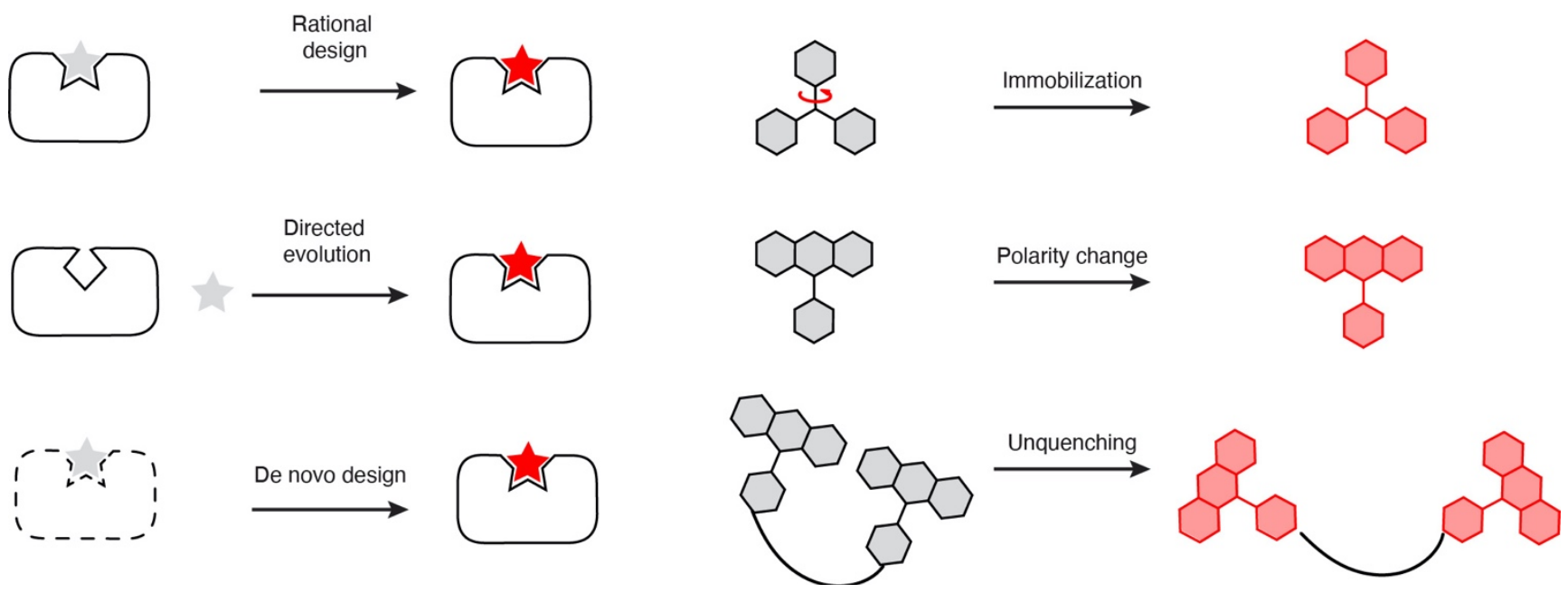

Figure 1. Tag and fluorogen engineering enable the generation of innovative fluorescent chemogenetic reporters.

scaffold by mimicking the strategy employed by Nature to create new functions. De novo design of proteins on the other hand is a powerful and attractive alternative, as it allows the building of proteins with predetermined structures and functions through exploration of the full sequence space in silico.

\subsubsection{Rational design}

The engineering of alternative fluorescent proteins from natural photoreceptor nicely illustrates the power of rational design (Figure 1). Photoreceptors allow organisms to use light as source of energy or as information to trigger a biological response. They include a prosthetic chromophore (e.g. flavins, bilins, retinal) that initiates a photochemical reaction upon light absorption. Most photoreceptors have evolved to maximize the efficiency of their photocycle, and thus barely fluoresce. However, introduction of variations in the backbone of photoreceptors can impair their natural photocycle and enhance their fluorescence emission.

This strategy allowed the development of fluorescent reporters from LOV (light, oxygen, voltage) sensing domains found in plants, algae and bacteria. Upon blue light excitation, a covalent adduct is formed in these flavoproteins between a flavin mononucleotide (FMN) chromophore and a conserved cysteine inducing a conformational change and thus a biological response [7]. Mutation of the conserved cysteine to alanine inhibits their natural photocycle and maximizes their fluorescence (Figure 2). This allowed the generation of flavin-based fluorescent proteins (FbFP) and iLOV from bacterial and plant LOV domains ${ }^{[8]}\left[{ }^{[9]}\right.$. FbFPs and iLOV fluoresce cyan-green light in response to blue light excitation thanks to the non-covalently bound FMN. Using comparative photobleaching measurements in $E$. coli colonies, a
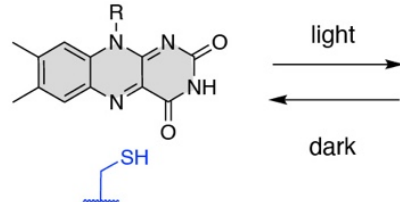<smiles>CCCN1NC2(C(=O)NC(=O)N=C2N)N(P)c2cc(C)c(C)cc21</smiles><smiles>[R]n1c2nc(=O)[nH]c(=O)c-2nc2cc(C)c(CN(C)C(=O)O)cc21</smiles>

Figure 2. Flavin-based fluorescent proteins (FbFPs) were engineered from LOV domains. Mutation of a conserved cysteine to alanine inhibits the natural photocycle and maximizes the fluorescence of FMN.

photostable iLOV variant was generated by directed evolution. Crystallographic analysis revealed that mutations close to the FMN-binding pocket rigidify the chromophore structure inside the cavity, providing greater resistance to photobleaching ${ }^{[10]}$. Thermostable FbFPs with superior photostability were also evolved from thermophilic bacteria ${ }^{[11]}$. Interestingly, as FMN is endogenously present and its biosynthesis does not require oxygen, FbFPs allowed the study of complex anaerobic processes in bacteria, fungi and mammalian cells ${ }^{[8]}{ }^{[12]}\left[{ }^{[13]}[14]\right.$. The shorter size of FbFPs and iLOV (ranging typically from 10 to 15 $\mathrm{kDa}$ ) is a clear advantage over GFP-like fluorescent proteins. The 
reduced genetic footprint of iLOV allowed for instance the study of virus infection and spread in plants ${ }^{[9]}$.

Rational design combined with random mutagenesis have been also essential in the engineering of far-red and near-infrared fluorescent proteins from phytochromes and their analogs. Phytochromes are present in algae, fungi, plant, bacteria and cyanobacteria. They sense far-red light through photoisomerization of a covalently attached biliverdin (BV) chromophore and function as light driven signal transducers ${ }^{[15]}$ Biliverdin is a catabolic product of heme and is endogenously present in cells. In a seminal paper, Tsien and co-workers showed that suppression of the PHY domain of the bacteriophytochrome of Deinococcus radiodurans and introduction of key mutations in the immediate environment of the chromophore in the remaining PAS-GAF domains prevented BV photoisomerization, locking it into an emissive conformation (Figure 3 ). The resulting fluorescent protein, named IFP1.4, fluoresces above $700 \mathrm{~nm}{ }^{[16]}$. Such system opened new exciting prospects for deep-tissue and whole-body imaging because of the higher transparency, lower light scattering, and lower autofluorescence of mammalian tissues in the near-infrared region. Massive engineering effort using rational design (but also directed evolution) has been done, using phytochromes from different organisms, to obtain far-red and near-infrared fluorescent proteins that are bright and photostable, incorporate efficiently BV and function as monomer [17][18] [19] [20]. The monomeric GAF domain of cyanobacteriochrome (CBRC) (found in cyanobacteria) was recently engineered to efficiently attach biliverdin (BV) rather than its usual phycocyanobilin (PCB) chromophore, giving eventually miRFP670nano, a small monomeric far-red fluorescent proteins of $17 \mathrm{kDa}$ that efficiently incorporates $\mathrm{BV}$ at endogenous levels [21].

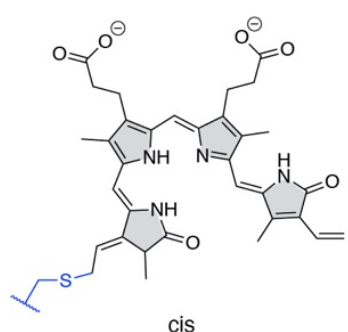

red state

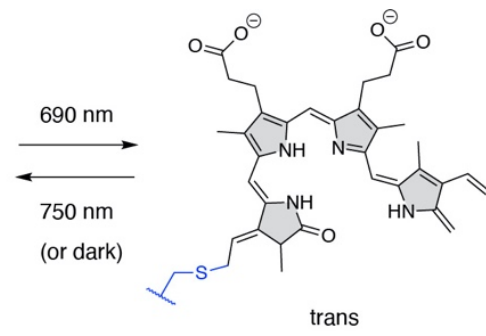

far-red state
Introduction of mutation stabilizing the fluorescent red state

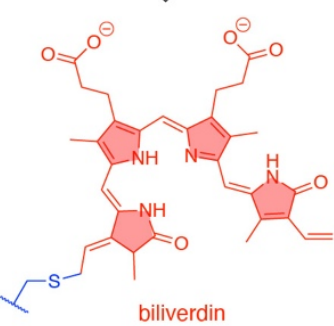

Figure 3. Far-red and near-infrared fluorescent proteins were engineered from phytochromes and their analogs. Introduction of key mutations prevents biliverdin photoisomerization, locking it into an emissive conformation.

Rational design can also be used elegantly to incorporate synthetic fluorogenic chromophores into natural protein scaffolds. The $15 \mathrm{kDa}$ cellular retinoic acid binding protein II CRABPII was engineered to incorporate a merocyanine dye precursor. This dye precursor exhibits no fluorescence when free and becomes fluorescent upon formation of a covalent adduct between its aldehyde group and a lysine residue rationally introduced in the chromophore binding site (Figure 4). The resulting iminiumcontaining merocyanine exhibits far-red emission with high quantum efficiency and brightness. Introduction of a large hydrophobic residue (Trp) and a negatively charge residue (Glu or Asp) close to the binding pocket improved the fluorescent quantum yield and brightness. Crystallographic structures of the protein:merocyanine complex revealed a relatively well packed and restricted chromophore within the binding cavity compared to the original protein ${ }^{[22]}$. For efficient use in mammalian and yeast cells, human cellular retinol-binding protein II (hCRBPII) was recently engineered in a similar fashion to covalently bind merocyanine aldehyde chromophore. Incorporation of a catalytic acid residue in the chromophore-binding pocket activates the precursor's carbonyl, favoring the nucleophilic attack of the reactive lysine residue ${ }^{[23]}$.

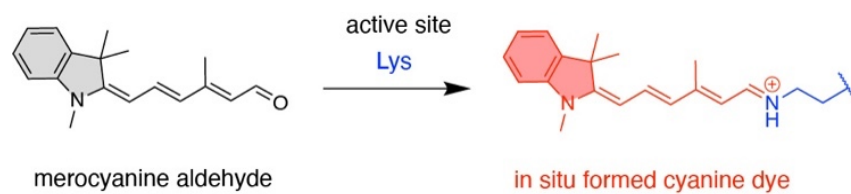

Figure 4. CRABPII was engineered to incorporate a merocyanine dye precursor, which exhibits no fluorescence when free and becomes fluorescent upon formation of a covalent adduct between its aldehyde group and a lysine residue rationally introduced in the chromophore binding site.

\subsubsection{Directed evolution}

When rational design cannot be used, directed evolution can be a very efficient way to create a complementary binding scaffold able to activate the fluorescence of a fluorogenic chromophore (Figure 1). A landmark study is the development of Fluorogen Activating Proteins (FAPs). FAPs are single chain antibodies (scFvs) able to bind non-covalently and activate the fluorescence of thiazole orange (TO) and malachite green (MG) derivatives (Figure 5), two molecular rotors known to dissipate light energy radiatively when conformationally immobilized [24] [25]. Human scFvs were chosen as binding scaffolds because of their relatively small size $(<30 \mathrm{kDa})$, their ability to recognize a wide range of antigens and for their ability to act as genetic fusion tags. FAPs were identified screening large yeast-displayed libraries of human scFvs by fluorescence activating cell sorting ${ }^{[26]}$. This strategy was expanded to several fluorogenic dimethylindole red (DIR) derivatives to identifying FAPs that cover most of the visible and near IR region of the spectrum ${ }^{[27]}$. The use of FAPs was originally restricted to image proteins at the cell surface or in non-reducing environments such as the secretory pathway, because scFv required the formation of conserved disulfide bonds for proper folding, stability and function. Directed evolution and activitybased selection allowed the development of a FAP variant containing a single point mutation that restored the activity lost in the disulfide-bond free mutant and that showed intracellular activity ${ }^{[28]}$. FAPs display reduced photobleaching because of fluorogen renewal, opening great prospects for live-cell superresolution microscopy. Highly photostable FAP has been successfully applied for tracking proteins in live bacterial cells using stimulated emission depletion (STED) microscopy ${ }^{[29]}$. FAPs also allowed single molecule tracking in live cells labeling only a subpopulation of proteins with low concentrations of fluorogen ${ }^{[30]}$.

Directed evolution using yeast display and fluorescence activated cell sorting is a general method for engineering fluorogen-activating scaffolds. Recently, this approach was 
employed to engineer the Fluorescence Activating and absorption Shifting Tag (FAST), a small protein of $14 \mathrm{kDa}$ that forms noncovalent fluorescent complexes with fluorogenic hydroxybenzylidene rhodanine (HBR) analogues ${ }^{[31]}$ (Figure 5). Composed of an electron-donating phenol ring conjugated with an electron-withdrawing rhodanine heterocycle, these push-pull fluorogens dissipate light energy non-radiatively in solution but strongly fluoresce when they are conformationally locked within the cavity of FAST. FAST was evolved from the Halorhodospira halophila Photoactive Yellow Protein (PYP), a natural photoreceptor sensing blue light through photoisomerization of a covalently attached hydroxycinnamoyl chromophore ${ }^{[32]}$. Taking advantage of the common structural features of PYP's natural chromophore and HBR analogs, PYP binding pocket was repurposed to bind HBR analogs. FAST allowed the selective labeling of fusion proteins in various localizations in living cells such as bacteria, yeast, mammalian cells and in multicellular organisms like zebrafish embryos ${ }^{[31]}$. Because of its oxygenindependence, FAST allowed the efficient imaging of proteins in strict anaerobe Clostridium species ${ }^{[33]}$ and the study of bacterial biofilm dynamics in low-oxygen environments ${ }^{[34]}$. Moreover, as fluorogens bind non-covalently, labeling can be easily reversed by washing, and fluorogen is continuously recycled reducing the apparent photobleaching rate ${ }^{[35]}$. As only a subset of proteins can be labeled, FAST can be used to image proteins below the diffraction limit using super-resolution imaging by radial fluctuations (SRRF) ${ }^{[36]}$, which is sensitive to fluorophore density, or by single-molecule localization microscopy (SMLM) ${ }^{[37]}$, which necessitates the stochastic generation of sparse subsets of emitters over time.

\section{A}

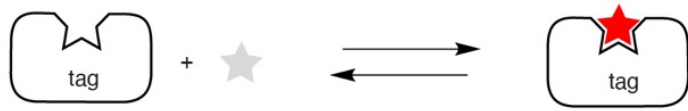

B
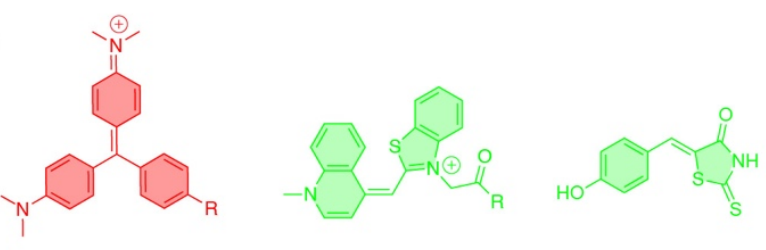

Malachite Green

Thiazole Orange

HBR

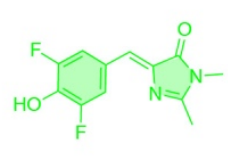

DFHBI

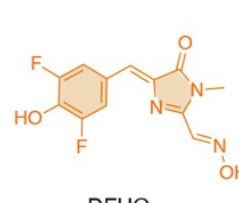

DFHO

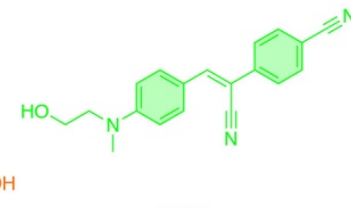

$\mathrm{HBC}$
Figure 5. Directed evolution can be used to generate $(A)$ complementary binding scaffolds able to activate the fluorescence of a fluorogen. (B) Examples of synthetic fluorogens.

Directed evolution was also essential for the development of RNA aptamers able to bind and activate the fluorescence of fluorogenic chromophores. Such aptamers open new ways to image RNA in live cells. The design of RNA aptamers binding selectively triphenylmethane dyes such as malachite green opened this field of research. Although these systems showed intrinsic cytotoxicity, making them unsuitable for in vivo imaging ${ }^{[38]}$, this first generation of RNA aptamers ${ }^{[38] ~[39] ~[40] ~}$ set up the basis for the design of effective fluorogenic RNA aptamers. In 2011, an RNA aptamer named Spinach was successfully designed to switch on the fluorescence of the 3,5-difluoro-4hydroxybenzylidene imidazolinone (DFHBI) ${ }^{[41]}$ (Figure 5). Mimick of the chromophore found in GFP, DFHBI is barely fluorescent in solution but exhibits fluorescence enhancement when encased into Spinach. Selected by systematic evolution of ligands by exponential enrichment (SELEX), Spinach is an RNA aptamer in which DFHBI chromophore is stacked, preventing its intramolecular motions, thus enhancing the fluorescence. In the original Spinach aptamer, the anionic form of the chromophore is embedded on top of a three-tetrad quadruplex forming the aptamer's core, which is stabilized by the presence of cations ${ }^{[42]}$ [43] [44]. Crystallographic studies have permitted to engineer variants with improved folding and thermal stability such as Spinach2 [45], iSpinach [46] and baby Spinach, a miniaturized Spinach aptamer [43]. Whereas the Spinach family was engineered on the basis of the affinity for DFHBI, SELEX coupled to fluorescence activated cell sorting (FACS) permitted to identify the Broccoli aptamer ${ }^{[47]}$. In presence of DFHBI analogues, Broccoli exhibited improved thermostability, robust folding and reduced magnesium dependence compared to Spinach, which overall increased its effective brightness ${ }^{[48]}$.

A similar strategy was employed for engineering a photostable small RNA aptamer called Corn that binds the 3,5difluoro-4-hydroxybenzylideneimidazolinone-2-oxime (DFHO) fluorophore, an analogue of the red fluorescent protein (RFP) chromophore (Figure 5). Corn enabled direct quantitative measurement of RNA levels when used as a RNA-based reporter for transcription ${ }^{[49]}$. Corn was shown however to form a dimer, which is the only form that binds DFHO, making it unsuitable for tagging and imaging mRNAs in cells.

The analogs of GFP or RFP chromophores are not the only fluorogens that have been used for the design of fluorogenic aptamers. Various other classes were used. Using stringent selection conditions, the RNA aptamer Mango was selected to bind Thiazole Orange derivatives with nanomolar affinities (Figure 5). The visualization of Mango in Caenorhabditis elegans gonads demonstrated its potential for live-cell RNA imaging [50]. Microfluidic droplet-based functional selection was performed to select Mango variants with improved fluorescent brightness [51]. SELEX was also recently used to select Pepper, an aptamer binding derivatives of HBC (4-((2-hydroxyethyl)(methyl)amino)benzylidene)-cyanophenylacetonitrile), synthetic dyes with structurally rigid electron acceptors and strong electron donors that strongly fluoresce upon constraining intramolecular motion [52] (Figure 5). Pepper allowed the study of intracellular noncoding RNAs and mRNAs and to label endogenous chromosomal loci. Furthermore, displaying high brightness and photostability, Pepper was shown to be well suited for live-cell imaging of RNA aptamers beyond the diffraction limit.

\subsubsection{In silico and de novo design}

Protein engineering can benefit from the use of in silico methods. In silico mutagenesis of residues within the ligand binding pocket of $E$. coli lipocalin (Blc) allowed computational screening of binders for a library of aminated GFP-like chromophores. The dynamic exchange of the fluorogen dye was shown to increase the photostability of the protein-fluorogen pairs. These systems allowed single molecule localization microscopy using low dye concentrations ${ }^{[53]}$. 
The design of fluorogen-activating proteins have recently taken a new turn with the use of de novo protein design, which facilitates and reduces the long process of protein engineering based on natural proteins ${ }^{[54]}$ (Figure 1). De novo design was used to create a small $\beta$-barrel which binds DFHBI, analog of the GFP chromophore (vide supra). Candidates were identified using computational screening method that combined a fixed backbone design (surrounding the fluorogen binding site) and a flexiblebackbone design (for the rest of the protein). Hierarchical gridbased search method allowed the screening of sequences, in which each single amino acid was randomly mutated, for fluorescence activation and proper folding in presence of DFHBI. Crystallographic structures revealed that stable monomeric variants of 110 amino acids could activate DFHBI fluorescence by stabilizing it in the binding pocket through multiple hydrogen bonds. Although they display weak brightness, these minifluorescence-activating proteins (mFAP) could be imaged in bacterial, yeast and mammalian cells by conventional widefield and confocal microscopy. mFAPs constitute the first generation of fluorogen-based reporters fully engineered by computational design, and considerable space for improvement remains possible. The use of de novo design for the generation of fluorogen-based reporters and biosensors is just in its infancy, and there is no doubt that computational approaches will make significant contributions in the future for the design of efficient reporters and biosensors ${ }^{[5]}$.

\subsection{Fluorogen engineering}

Semi-synthetic hybrid systems being composed of a synthetic fluorogenic part, it is possible to benefit from the power of molecular engineering to create, tune or optimize hybrid fluorescent reporters. High contrast imaging requires both high fluorogenicity and selective activation, two parameters that can be engineered using modern organic chemistry (Figure 1). Molecular engineering offers also new possibilities for tuning various properties such as cellular uptake or spectral characteristics.

\subsubsection{Design}

In the systems described so far, fluorogenicity arose from immobilization of molecular rotors or push-pull systems (Figures 2-5). These fluorogens are essentially non-fluorescent in solution because they dissipate light energy through efficient non-radiative processes. However, when locked in a planar conformation in a well fitted cavity, these processes are slow down and fluorescence becomes an efficient energy dissipation mechanism These systems are however not the only ones that can be used to generate fluorogenic hybrid systems. Other mechanisms can be used to switch on fluorescence through molecular interaction.

The development of fluorogenic rhodamine derivatives nicely illustrates how molecular engineering allows the fine tuning of fluorogenicity. Rhodamines can exist as a non-fluorescent spirolactone closed form or as a fluorescent zwitterionic opened form (Figure 6). Creatively exploited to design photoactivatable and blinking fluorescent dyes for super-resolution imaging ${ }^{[56][57][58]}$ this unique property can be used for the design of fluorogenic rhodamines. In particular silicon rhodamines were shown to exist mainly in their non-fluorescent spirolactone closed form in aqueous solution, but to adopt the fluorescent zwitterionic opened form (that displays excitation and emission maxima in the far-red and near-infrared region) in less polar environments. This property allowed the development of a collection of fluorogenic substrates for protein labeling, exploiting the fact that a protein environment is less polar than aqueous solution. SiR derivatives were coupled to substrates for self-labeling tags such as SNAPtag, CLIP-tag and HaloTag [59] [60] [61], allowing fluorogenic labeling of fusion proteins in live cells (Figure 6). SiR fluorophores have been shown to be well suited for protein labeling in cells and multicellular organisms, multi-color imaging, and super-resolution imaging. RNA aptamers were evolved to recognize SiR and photostable carborhodamine fluorophores with nanomolar affinities. Thanks to their far-red shifted fluorescence, these RNA aptamers were successfully applied to visualize mRNAs in live bacteria using STED microscopy ${ }^{[62]}$. The use of four-membered azetidine ring allowed furthermore the generation of fluorogenic rhodamine analogs with higher photostability and brightness [63][64] The lactone-zwitterion equilibrium constant enables to predict the fluorogenicity of rhodamines, providing a quantitative framework for the design of new fluorogenic dyes with optimal fluorogenicity ${ }^{[58]}$. Recently, a general method has been developed to transform regular rhodamine and related fluorophores into fluorogenic chromophores with an excellent cell-permeability. Converting their carboxyl group into an electron-deficient amide enabled to tune the dynamic equilibrium between the fluorescent zwitterion and the non-fluorescent, cell-permeable spirolactam, allowing the generation of fluorogens that light up upon interaction with their target. Using this design, fluorogens with various spectral properties could be designed for wash-free, multi-color, live cell nanoscopy ${ }^{[65]}$.
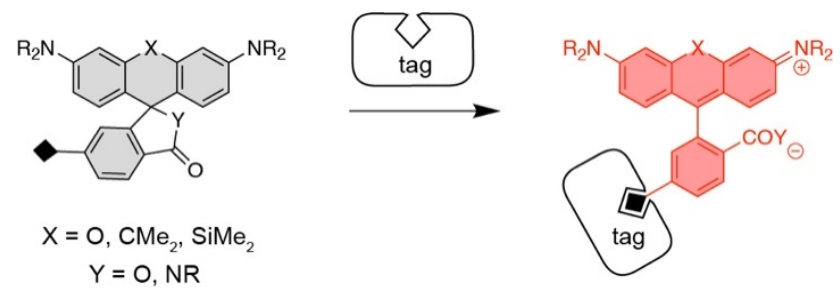

Figure 6. Fluorogenic rhodamines. Rhodamines can exist mainly in their nonfluorescent spirolactone closed form in aqueous solution, but adopt their fluorescent zwitterionic opened form when bound to their target tag.

Fluorogenic substrate were also designed relying on intramolecular quenching. This was originally proposed for the labeling of the PYP-tag, which can react in a covalent fashion with hydroxycinnamoyl and coumarin thioester derivatives. Fluorogenicity was obtained using substrates composed of a fluorophore linked to a quencher. Reaction with PYP leads to fluorophore unquenching and restores fluorescence ${ }^{[66][67][68]}$. The approach is versatile and allowed one to change the spectral properties by changing the fluorophore. Multicolor imaging using this approach enabled to elucidate the role of the GLUT4 glycosylation in living cells ${ }^{[69]}$. For efficient use in live cells, highly cell-permeant and reactive substrates were designed using smaller polarity-sensitive coumarins ${ }^{[70]}$. In highly polar environment, coumarin ligand adopts a twisted intramolecular charge transfer (TICT) non-fluorescent state. Whereas in lower polar environment, as PYP's binding pocket, the planar conformation of the probe induced a high fluorescence exaltation. A combined mutational and chemical approach was used to create electrostatic interactions between the substrate and the tag, further increasing the temporal resolution of the labeling and the brightness ${ }^{[71]}$. Introduction of a hydroxyl group into coumarin ligand enabled to increase the labeling reaction rate and the OFFON fluorescence ratio ${ }^{[72]}$. This probe allowed the observation of the transient nuclear localization of the mitochondrial deacetylase sirtuin-3. 

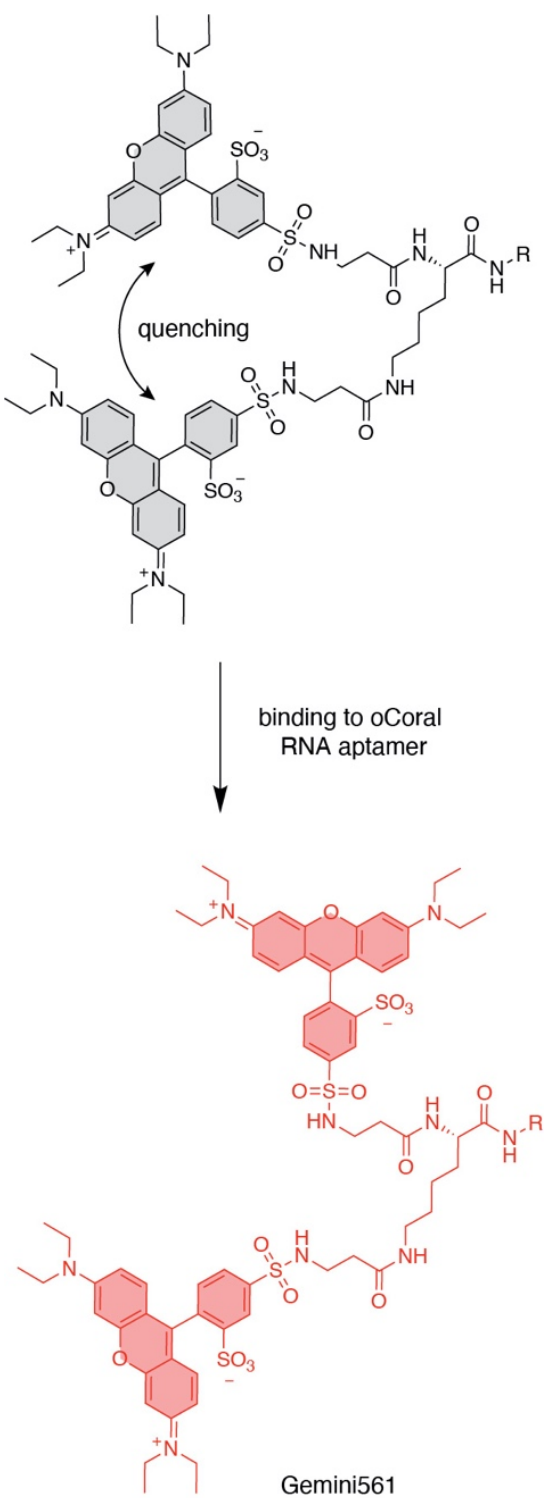

Figure 7. Example of fluorogens based on intramolecular unquenching. Gemini561 is a fluorogen composed of two molecules of sulforhodamine B that self-quench in solution but strongly fluoresce when they interact with the RNA aptamer oCoral.

Intramolecular quenching was also creatively used for the design of fluorogenic probes for RNA labeling. The aptamer Riboglow was developed from a short riboswitch sequence that recognizes cobalamin ${ }^{[73]}$. Cobalamin can efficiently quench an attached fluorophore. Binding to the riboswitch shields the fluorophore from cobalamin, resulting thus in high fluorescence activation. Riboglow allowed the efficient imaging of RNA Pol II transcripts. Similarly, was developed Gemini561, a fluorogen composed of two molecules of sulforhodamine $B$ that self-quench in solution but strongly fluoresce when they interact with the RNA aptamer oCoral (Figure 7). This ultrabright aptamer, selected by SELEX and microfluidic-assisted in vitro compartmentalization, was shown to display unprecedented photostability compared to Broccoli, Mangolll and Corn aptamers ${ }^{[74]}$.

\subsubsection{Tuning}

Molecular engineering can also creatively be used to tune fluorogen properties, such as cellular uptake. Although highly membrane-permeant fluorogens are preferred for efficient intracellular protein labeling, membrane-impermeant fluorogens can restrict labeling to proteins located at the cell surface. The use of membrane-impermeant fluorogens allowed the monitoring of dynamic processes such as membrane protein trafficking, internalization, recycling or endocytosis. Membrane impermeant malachite green (MG) derivatives enabled for instance to selectively track FAP-tagged membrane receptors at the cell surface and to investigate the role of G-protein coupled receptors trafficking in live cells. Addition of amphiphilic polyethyleneglycol linkers or negatively charged sulfonate groups reduces their cellular uptake (Figure 8), limiting their action to the plasma membrane only ${ }^{[75]}[76]$. A sulfonated $M G$ analog combined with a FAP-tagged anti-EGFR affibody was recently used for tumor visualization in mice ${ }^{[7]}$. Similarly, membrane impermeant HBR derivatives containing a negatively charged carboxymethyl group on the rhodanine head allowed the selective labeling of FASTtagged proteins at the cell surface (Figure 8), enabling to easily quantify the levels of cell-surface proteins by various fluorometric techniques ${ }^{[78]}$.

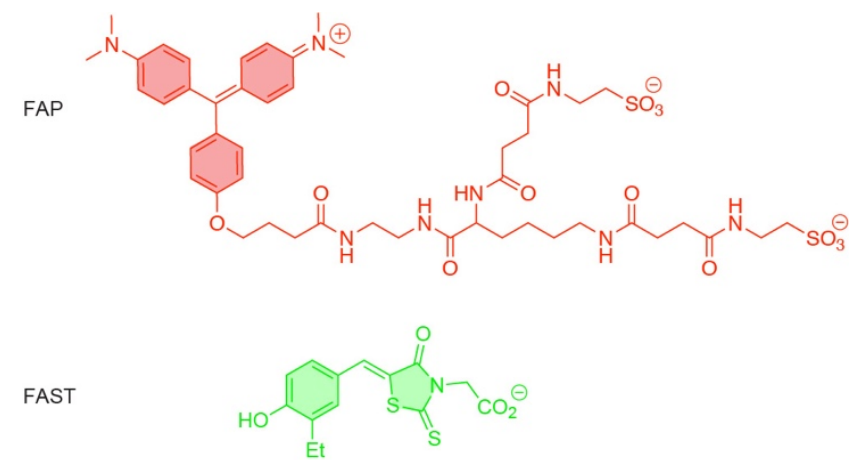

Figure 8. Membrane-impermeant fluorogens for FAP and FAST were generated by addition of negatively charged groups.

Molecular engineering is also efficient in tuning the spectral properties of fluorogens. Structural modifications can induce significant changes in the electronic properties of fluorogens, and thus their absorption and emission properties. The spectral properties of many fluorogenic reporters have been successfully tuned by molecular engineering, allowing with a single tag to have several colors, providing a versatility that is not encountered with fluorescent proteins. The use of electron-donating thiophene aryl groups has permitted to extend the spectral properties of FAP:MG analogs to the red ${ }^{[79]}$. Introduction of electron-donating groups on the aromatic ring of HBR analogs allowed the design of FAST fluorogens with red-shifted absorption and emission properties for applications in multicolor imaging ${ }^{[80]}$. The ability to change color by fluorogen exchange allowed dynamic color switching in cells, providing a unique kinetic signature that could be advantageously exploited for highly multiplexed imaging ${ }^{[00]}$. By extending the length of the conjugated $\pi$-system, in addition to the introduction of electron-withdrawing or donating heteroatom groups, a panel of GFP-like aminated chromophores derivatives enabled to red shift the spectral properties of the lipocalin-based system ${ }^{[81]}$. The spectral properties of Spinach could be tuned by chemical modification of GFP-like chromophores. Substitutions on the imidazolone ring of DFHBI permitted to optimize the fluorescence of the Spinach RNA and its derivatives to the optical filters commonly used in fluorescence microscopy [82]. Molecular engineering also enabled to tune the spectral properties of the RNA aptamer Pepper from cyan to red by tuning the aromatic $\pi$ structure and adjusting the electron donor/acceptor capability of 
the dye ${ }^{[52]}$. Similarly, the spectral properties of rhodamine-based fluorogens could be varied by changing the bridge in the xanthene moiety [64] [65]. Finally, molecular engineering can extend the spectral and photophysical properties of natural fluorogens. Computational studies based on FMN chromophore predicted theoretically the structures of derivatives with red-shifted emission of fluorescence covering spectral regions of 500 up to $860 \mathrm{~nm}{ }^{[83]}$.

\section{Hybrid actuators and biosensors}

\subsection{Actuators}

Beyond offering new opportunities in biological imaging, the development of chemogenetic hybrid systems opens also new prospects for the design of actuators enabling to study biological systems in new ways. A nice illustration of the potential of chemogenetic hybrids is the development of reactive oxygen species (ROS) producers. ROS are known to cause oxidative damage to DNA, lipids and proteins in living cells. They are naturally involved in many signaling processes (e.g. autophagy, apoptosis and aging). ROS generators can be used for photoinducible protein inactivation or cell ablation, in photodynamic therapy for targeting cancer cell lines or in electron microscopy (EM) for local synthesis of osmiophilic products resolvable by EM. A genetically encoded photosensitizer was engineered from the LOV2 domain of Arabidopsis thaliana phototropin 2, which naturally binds FMN (vide supra). Directed evolution using IFP1.4 photobleaching as an indicator for ROS generation allowed the generation of the mini Singlet Oxygen Generator (miniSOG). Crystallographic studies showed an increase in rigidity of the chromophore's environment compared to wild-type LOV2 suggesting that oxygen diffusion can occur properly ${ }^{[84]}$. When irradiated with blue light, miniSOG fluoresces and generates both superoxide anion (and its derivatives) and singlet oxygen $\left({ }^{1} \mathrm{O}_{2}\right)$ through type I and II mechanisms ${ }^{[85]}\left[{ }^{[86]}\right.$. This dual function allowed the imaging of MiniSOG by correlative light and electron microscopy (CLEM) in Caenorhabditis elegans and mice synaptic cells ${ }^{[87]}$. Protein engineering enabled to enhance ${ }^{1} \mathrm{O}_{2}$ generation by mutating residues responsible for hydrogen bonds and electron-donating transfer between the chromophore and the protein scaffold ${ }^{[88]}{ }^{809}$. Engineered MiniSOG variant with improved ROS generation has been successfully used to induce specific cell ablation in Drosophila melanogaster adult fly ${ }^{[00]}$.

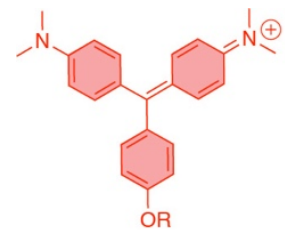

fluorogen

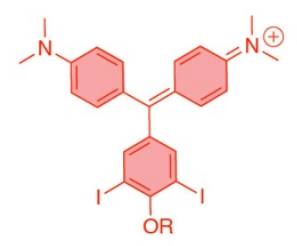

fluorogen \& ROS generator
Figure 9. Targeted and Activated Photosensitizer (TAP). Malachite Green with heavy iodine atoms forms with FAP/TAP a complex producing ROS because of increased intersystem crossing.

A far-red fluorogenic photosensitizer was recently developed from the FAP family for applications in deep tissues. Malachite green was substituted with heavy iodine atoms to increase intersystem crossing and thus ROS production (Figure 9). The resulted system FAP-TAPs (Targeted and Activated Photosensitizer) fluoresces and produces singlet oxygen only when the exogenous dye is associated to FAP. Due to the very short excited state lifetime of free fluorogen, reactive oxygen species appear exclusively upon binding to the encasing protein which makes the system a unique 'on-demand' genetically encoded photosensitizer. This system allowed deep cell ablation in adult zebrafish and subcellular protein inactivation in living cells [91].

\subsection{Biosensors}

As alternative to fluorescent proteins, fluorescent chemogenetic reporters can be creatively used for the design of various types of biosensors enabling to visualize intracellular events in living cells with high resolution in space and time. Biosensors can be generated by taking advantage of the intrinsic advantages of fluorescent chemogenetic reporters.

As all GFP-like fluorescent proteins depend on the presence of $\mathrm{O}_{2}$ for chromophore maturation, $\mathrm{O}_{2}$-insensitive fluorogenic systems allow the creation of genetically encoded biosensors measuring $\mathrm{O}_{2}$ levels in living cells. The oxygen-insensitive FbFP allowed the design of a FRET-based oxygen biosensor named FluBO. Oxygen variation was monitored in real-time in $E$. coli by measuring the FRET efficiency between FbFP donor and the hypoxia-sensitive yellow fluorescent protein (YFP) [92]. Reoxygenation of cells was also visualized by controlling the expression of the oxygen-insensitive UnaG with an hypoxia responsive promoter ${ }^{[3]}$. Addition of a disulfide bridge to the $\beta$ barrel surface of UnaG allowed moreover the generation of a redox-sensitive fluorescent sensor with high dynamic range, enabling to monitor redox changes under hypoxia in living cells [94].

Modification of the fluorogenic chromophore through molecular engineering is another way to generate biosensors. FAPs were for example used to create a surface-selective $\mathrm{pH}$ biosensor using $\mathrm{pH}$-sensitive cyanine analogues covalently attached to thiazole orange or malachite green. Change in FRET efficiency induced by $\mathrm{pH}$ change allowed the study of receptormediated endocytosis or receptor recycling ${ }^{[95]}$ [96]. A ratiometric $\mathrm{pH}$ sensor was also engineered from hCRBPIl using a nonpermeant julolidine retinal analog. The presence of a titratable functional group close to the chromophore binding pocket renders both the absorption and fluorescence emission sensitive to $\mathrm{pH}$ change ${ }^{[97]}$.

Fluorescent chemogenetic reporters can also be used creatively to generate new types of biosensors. By coupling chemogenetic reporters with a sensing domain, it is possible to condition the fluorogen binding (and thus fluorescence) to the recognition of the analyte. Insertion of Calmodulin (CaM), a wellestablished $\mathrm{Ca}^{2+}$ sensing domain, within UnaG permitted to develop BReleaCa, a calcium dual-ligand fluorogenic biosensor. $B R e l e a C a$ releases bilirubin (BR) upon calcium binding, leading to a loss of fluorescence ${ }^{[98]}$. $\mathrm{A} \mathrm{Ca}^{2+}$ biosensor with a more favorable positive response was developed coupling a circularly permuted version of FAST to CaM and the CaM-binding peptide of myosin light-chain kinase (M13). In this sensor, calcium binding promotes fluorogen binding, and thus fluorescence increase, enabling to image $\mathrm{Ca}^{2+}$ levels in cells ${ }^{[99]}$. Sensors can also be built by conditioning the fluorogen binding to a given signal. An infrared fluorescent protease reporter was engineered from a circularly permuted IFP1.4 so that the biliverdin chromophore incorporation is regulated by protease activity. The 'inactivated' sensor iProtease is not fluorescent because of a hidden cysteine residue essential for BV attachment. Proteolytic activation frees the cysteine residue which returns to the binding cavity, thus 
promoting fluorescence by proper BV incorporation. This system allowed the development of iCasper, an apoptosis cellular sensor which fluoresces under the activation of caspase-3 [100].

Fluorescent chemogenetic reporters offer also great opportunities for the design of split fluorescent reporters for the detection of protein-protein interactions (PPIs). The protein module can be split into two complementary modules, which can assemble when fused to two interacting proteins, promoting fluorogen binding and thus fluorescence activation. Such complementation system was designed by splitting FAST in two complementary fragments. The resulting splitFAST was shown to allow the visualization of PPIs in living cells. The complementation of splitFAST was demonstrated to be rapid and reversible, enabling to study protein complex association and dissociation almost in real-time, which has not been achievable using splitGFP derivatives ${ }^{[101]}$. Other fluorogenic split systems working on the same principle were engineered from UnaG ${ }^{[102]}$ and the biliverdinbased far-red fluorescent proteins [103] [104] [105]. Recently, a split version of miniSOG was designed to track PPIs by electron microscopy (EM). Protein-protein interactions allowed the generation of reactive oxygen species from split-miniSOG which in combination with diaminobenzidine produced a localized polymeric precipitate providing high contrast in EM in presence of osmium [106]

\section{Conclusion}

Hybrid reporters have recently opened new ways to image living cells and sense biochemical events. Benefiting from numerous assets, as their shorter size, their $\mathrm{O}_{2}$ independence, their ondemand fluorescence, fluorogenic systems present advantages over the widely used autofluorescent proteins.

As hybrid reporters are composed of a protein or an RNA tag and a fluorogenic chromophore, the development and improvement of such systems can be done through engineering both components. Engineering the scaffold by rational design and directed evolution permit to generate scaffolds able to activate the fluorescence of natural or synthetic fluorogens and significantly enhance their performance. The use of de novo design opens more over great prospect for further development and/or improvement of custom fluorogenic systems. By reducing the duration of the long-process of directed evolution (and other related methods), de novo design represents a promising way to design fluorogenic reporters from scratch. Moreover, de novo design should greatly facilitate the development of chemogenetic biosensors by speeding up the initial design and reducing the duration of the optimization steps.

The possibility to create, tune or optimize hybrid chemogenetic reporters through molecular engineering of the fluorogenic chromophores represents moreover a major advantage over fluorescent proteins. While fluorescent proteins cannot be tuned as desired, as their fluorescence is restricted to their embedded genetically encoded chromophore, the use of synthetic fluorogens permits to finely tune the properties of chemogenetic systems. Various fluorescence activation mechanisms can be used, and a broad palette of colors can be obtained. Beyond creating fluorogens with various photophysical properties, molecular engineering can also be used to increase or reduce fluorogen cellular uptake or to turn fluorogens into ROS generators for various applications in cell biology.

The development and use of fluorogenic reporters and biosensors is still in its infancy. We are convinced that hybrid systems will allow new discoveries in Biology from fundamental mechanisms to the causes of disease, and the development of novel diagnostic tools and therapeutics. There is no doubt that the continuous development of innovative and sophisticated imaging techniques (e.g. tissue clearing, expansion microscopy) and modalities (e.g. correlative light and electron microscopy, photoacoustic imaging) will motivate developers to invent fully adapted chemogenetic reporters and biosensors in order to make the most of these technologies and push forward the frontiers of biological imaging.

\section{Notes}

The authors declare the following competing financial interest: A.G. is co-founder and hold equity in Twinkle Bioscience / The Twinkle Factory, a company commercializing the FAST technology.

\section{Acknowledgements}

This work was supported by the European Research Council (ERC-2016-CoG-724705 FLUOSWITCH).

Keywords: Fluorescent labels and biosensors • chemogenetic reporters • fluorescence imaging

[1] R. Y. Tsien, Annu. Rev. Biochem. 1998, 67, 509-544

[2] R. Y. Tsien, FEBS Lett. 2005, 579, 927-932.

[3] B. T. Bajar, E. S. Wang, A. J. Lam, B. B. Kim, C. L. Jacobs, E. S. Howe, M. W. Davidson, M. Z. Lin, J. Chu, Sci. Rep. 2016, 6, 20889.

[4] N. C. Shaner, G. G. Lambert, A. Chammas, Y. Ni, P. J. Cranfill, M. A. Baird, B. R. Sell, J. R. Allen, R. N. Day, M. Israelsson, et al., Nat. Methods 2013, 10, 407-409.

[5] D. M. Chudakov, M. V. Matz, S. Lukyanov, K. A. Lukyanov, Physiol. Rev. 2010, 90, 1103-1163.

[6] A. Kumagai, R. Ando, H. Miyatake, P. Greimel, T. Kobayashi, Y. Hirabayashi, T. Shimogori, A. Miyawaki, Cell 2013, 153, 1602-1611.

[7] A. Losi, E. Polverini, B. Quest, W. Gärtner, Biophys. J. 2002, 82, 26272634.

[8] T. Drepper, T. Eggert, F. Circolone, A. Heck, U. Krauss, J.-K. Guterl, M. Wendorff, A. Losi, W. Gärtner, K.-E. Jaeger, Nat. Biotechnol. 2007, 25 , 443-445.

[9] S. Chapman, C. Faulkner, E. Kaiserli, C. Garcia-Mata, E. I. Savenkov, A. G. Roberts, K. J. Oparka, J. M. Christie, Proc. Natl. Acad. Sci. U. S. A. 2008, 105, 20038-20043.

[10] J. M. Christie, K. Hitomi, A. S. Arvai, K. A. Hartfield, M. Mettlen, A. J. Pratt, J. A. Tainer, E. D. Getzoff, J. Biol. Chem. 2012, 287, 22295-22304.

[11] M. Wingen, K.-E. Jaeger, T. Gensch, T. Drepper, Photochem. Photobiol. 2017, 93, 849-856.

[12] T. Drepper, T. Gensch, M. Pohl, Photochem. Photobiol. Sci. 2013, 12, 1125-1134.

[13] A. Mukherjee, C. M. Schroeder, Curr. Opin. Biotechnol. 2015, 31, 16-23.

[14] M. Wingen, J. Potzkei, S. Endres, G. Casini, C. Rupprecht, C. Fahlke, U. Krauss, K.-E. Jaeger, T. Drepper, T. Gensch, Photochem. Photobiol. Sci. 2014, 13, 875-883.

[15] M. Baloban, D. M. Shcherbakova, S. Pletnev, V. Z. Pletnev, J. C. Lagarias, V. V. Verkhusha, Chem. Sci. 2017, 8, 4546-4557.

[16] X. Shu, A. Royant, M. Z. Lin, T. A. Aguilera, V. Lev-Ram, P. A. Steinbach, R. Y. Tsien, Science 2009, 324, 804-807.

[17] G. S. Filonov, K. D. Piatkevich, L.-M. Ting, J. Zhang, K. Kim, V. V. Verkhusha, Nat. Biotechnol. 2011, 29, 757-761.

[18] D. M. Shcherbakova, V. V. Verkhusha, Nat. Methods 2013, 10, 751-754. 
[19] D. Yu, W. C. Gustafson, C. Han, C. Lafaye, M. Noirclerc-Savoye, W.-P Ge, D. A. Thayer, H. Huang, T. B. Kornberg, A. Royant, et al., Nat Commun. 2014, 5, 3626.

[20] E. A. Rodriguez, G. N. Tran, L. A. Gross, J. L. Crisp, X. Shu, J. Y. Lin, R. Y. Tsien, Nat. Methods 2016, 13, 763-769.

[21] O. S. Oliinyk, A. A. Shemetov, S. Pletnev, D. M. Shcherbakova, V. V. Verkhusha, Nat. Commun. 2019, 10, 279.

[22] I. Yapici, K. S. S. Lee, T. Berbasova, M. Nosrati, X. Jia, C. Vasileiou, W. Wang, E. M. Santos, J. H. Geiger, B. Borhan, J. Am. Chem. Soc. 2015 137, 1073-1080

[23] E. M. Santos, T. Berbasova, W. Wang, R. E. Salmani, W. Sheng, C. Vasileiou, J. H. Geiger, B. Borhan, ChemBioChem 2020, 21, 723-729.

[24] J. Nygren, N. Svanvik, M. Kubista, Biopolymers 1998, 46, 39-51.

[25] J. R. Babendure, S. R. Adams, R. Y. Tsien, J. Am. Chem. Soc. 2003 125, 14716-14717.

[26] C. Szent-Gyorgyi, B. F. Schmidt, Y. Creeger, G. W. Fisher, K. L. Zakel, S. Adler, J. A. J. Fitzpatrick, C. A. Woolford, Q. Yan, K. V. Vasilev, et al., Nat. Biotechnol. 2008, 26, 235-240.

[27] H. Özhalici-Ünal, C. L. Pow, S. A. Marks, L. D. Jesper, G. L. Silva, N. I. Shank, E. W. Jones, J. M. Burnette, P. B. Berget, B. A. Armitage, J. Am Chem. Soc. 2008, 130, 12620-12621.

[28] B. P. Yates, M. A. Peck, P. B. Berget, Mol. Biotechnol. 2013, 54, 829841.

[29] S. Saurabh, A. M. Perez, C. J. Comerci, L. Shapiro, W. E. Moerner, J. Am. Chem. Soc. 2016, 138, 10398-10401.

[30] Q. Yan, S. L. Schwartz, S. Maji, F. Huang, C. Szent-Gyorgyi, D. S. Lidke K. A. Lidke, M. P. Bruchez, ChemPhysChem 2014, 15, 687-695.

[31] M.-A. Plamont, E. Billon-Denis, S. Maurin, C. Gauron, F. M. Pimenta, C. G. Specht, J. Shi, J. Quérard, B. Pan, J. Rossignol, Proc. Natl. Acad. Sci. 2016, 113, 497-502.

[32] R. Brudler, T. E. Meyer, U. K. Genick, S. Devanathan, T. T. Woo, D. P. Millar, K. Gerwert, M. A. Cusanovich, G. Tollin, E. D. Getzoff, Biochemistry 2000, 39, 13478-13486.

[33] H. E. Streett, K. M. Kalis, E. T. Papoutsakis, Appl. Environ. Microbiol. 2019, 85, e00622-19.

[34] A. Monmeyran, P. Thomen, H. Jonquière, F. Sureau, C. Li, M.-A Plamont, C. Douarche, J.-F. Casella, A. Gautier, N. Henry, Sci. Rep. 2018, 8, 10336

[35] F. M. Pimenta, G. Chiappetta, T. Le Saux, J. Vinh, L. Jullien, A. Gautier Sci. Rep. 2017, 7, 12316

[36] E. M. Smith, A. Gautier, E. M. Puchner, ACS Chem. Biol. 2019, 14, 11151120.

[37] M. Venkatachalapathy, V. Belapurkar, M. Jose, A. Gautier, D. Nair, Nanoscale 2019, 11, 3626-3632.

[38] J. R. Babendure, S. R. Adams, R. Y. Tsien, J. Am. Chem. Soc. 2003, 125, 14716-14717.

[39] M. N. Stojanovic, D. M. Kolpashchikov, J. Am. Chem. Soc. 2004, 126, 9266-9270.

[40] B. A. Sparano, K. Koide, J. Am. Chem. Soc. 2005, 127, 14954-14955.

[41] J. S. Paige, K. Y. Wu, S. R. Jaffrey, Science 2011, 333, 642-646.

[42] H. Huang, N. B. Suslov, N.-S. Li, S. A. Shelke, M. E. Evans, Y. Koldobskaya, P. A. Rice, J. A. Piccirilli, Nat. Chem. Biol. 2014, 10, 686691.

[43] K. D. Warner, M. C. Chen, W. Song, R. L. Strack, A. Thorn, S. R. Jaffrey, A. R. Ferré-D’Amaré, Nat. Struct. Mol. Biol. 2014, 21, 658-663.

[44] L. Truong, A. R. Ferré-D'Amaré, Protein Sci. 2019, 28, 1374-1386.

[45] R. L. Strack, M. D. Disney, S. R. Jaffrey, Nat. Methods 2013, 10, 1219 1224 .

[46] A. Autour, E. Westhof, M. Ryckelynck, Nucleic Acids Res. 2016, 44 2491-2500

[47] G. S. Filonov, J. D. Moon, N. Svensen, S. R. Jaffrey, J. Am. Chem. Soc 2014, 136, 16299-16308.

[48] G. S. Filonov, C. W. Kam, W. Song, S. R. Jaffrey, Chem. Biol. 2015, 22, 649-660.

[49] W. Song, G. S. Filonov, H. Kim, M. Hirsch, X. Li, J. D. Moon, S. R. Jaffrey, Nat. Chem. Biol. 2017, 13, 1187-1194.

[50] E. V. Dolgosheina, S. C. Y. Jeng, S. S. S. Panchapakesan, R. Cojocaru, P. S. K. Chen, P. D. Wilson, N. Hawkins, P. A. Wiggins, P. J. Unrau, ACS Chem. Biol. 2014, 9, 2412-2420.
[51] R. J. Trachman, A. Autour, S. C. Y. Jeng, A. Abdolahzadeh, A. Andreoni, R. Cojocaru, R. Garipov, E. V. Dolgosheina, J. R. Knutson, M. Ryckelynck, et al., Nat. Chem. Biol. 2019, 15, 472-479.

[52] X. Chen, D. Zhang, N. Su, B. Bao, X. Xie, F. Zuo, L. Yang, H. Wang, L. Jiang, Q. Lin, et al., Nat. Biotechnol. 2019, 1287-1293.

[53] N. G. Bozhanova, M. S. Baranov, N. V. Klementieva, K. S. Sarkisyan, A. S. Gavrikov, I. V. Yampolsky, E. V. Zagaynova, S. A. Lukyanov, K. A Lukyanov, A. S. Mishin, Chem. Sci. 2017, 8, 7138-7142.

[54] P.-S. Huang, S. E. Boyken, D. Baker, Nature 2016, 537, 320-327.

[55] J. Dou, A. A. Vorobieva, W. Sheffler, L. A. Doyle, H. Park, M. J. Bick, B Mao, G. W. Foight, M. Y. Lee, L. A. Gagnon, et al., Nature 2018, 561, 485-491.

[56] J. B. Grimm, B. P. English, H. Choi, A. K. Muthusamy, B. P. Mehl, P. Dong, T. A. Brown, J. Lippincott-Schwartz, Z. Liu, T. Lionnet, et al., Nat Methods 2016, 13, 985-988.

[57] S. Uno, M. Kamiya, T. Yoshihara, K. Sugawara, K. Okabe, M. C. Tarhan, H. Fujita, T. Funatsu, Y. Okada, S. Tobita, et al., Nat. Chem. 2014, 6, 681-689.

[58] Q. Zheng, A. X. Ayala, I. Chung, A. V. Weigel, A. Ranjan, N. Falco, J. B. Grimm, A. N. Tkachuk, C. Wu, J. Lippincott-Schwartz, et al., ACS Cent. Sci. 2019, 5, 1602-1613.

[59] G. Lukinavičius, K. Umezawa, N. Olivier, A. Honigmann, G. Yang, T. Plass, V. Mueller, L. Reymond, I. R. Corrêa Jr, Z.-G. Luo, et al., Nat. Chem. 2013, 5, 132-139.

[60] G. Lukinavičius, L. Reymond, E. D'Este, A. Masharina, F. Göttfert, H. Ta, A. Güther, M. Fournier, S. Rizzo, H. Waldmann, et al., Nat. Methods 2014, 11, 731-733.

[61] G. Lukinavičius, L. Reymond, K. Umezawa, O. Sallin, E. D'Este, F. Göttfert, H. Ta, S. W. Hell, Y. Urano, K. Johnsson, J. Am. Chem. Soc. 2016, 138, 9365-9368.

[62] R. Wirth, P. Gao, G. U. Nienhaus, M. Sunbul, A. Jäschke, J. Am. Chem. Soc. 2019, 141, 7562-7571.

[63] J. B. Grimm, B. P. English, J. Chen, J. P. Slaughter, Z. Zhang, A Revyakin, R. Patel, J. J. Macklin, D. Normanno, R. H. Singer, et al., Nat. Methods 2015, 12, 244-250

[64] J. B. Grimm, A. K. Muthusamy, Y. Liang, T. A. Brown, W. C. Lemon, R. Patel, R. Lu, J. J. Macklin, P. J. Keller, N. Ji, et al., Nat. Methods 2017 14, 987-994.

[65] L. Wang, M. Tran, E. D’Este, J. Roberti, B. Koch, L. Xue, K. Johnsson, Nat. Chem. 2020, 12, 165-172.

[66] Y. Hori, H. Ueno, S. Mizukami, K. Kikuchi, J. Am. Chem. Soc. 2009, 131, 16610-16611.

[67] Y. Hori, S. Hirayama, K. Kikuchi, Phil Trans R Soc A 2017, 375, 20170018.

[68] Y. Hori, K. Nakaki, M. Sato, S. Mizukami, K. Kikuchi, Angew. Chem. Int Ed. 2012, 51, 5611-5614

[69] S. Hirayama, Y. Hori, Z. Benedek, T. Suzuki, K. Kikuchi, Nat. Chem. Biol. 2016, 12, 853-859.

[70] Y. Hori, T. Norinobu, M. Sato, K. Arita, M. Shirakawa, K. Kikuchi, J. Am Chem. Soc. 2013, 135, 12360-12365.

[71] Y. Hori, S. Hirayama, M. Sato, K. Kikuchi, Angew. Chem. Int. Ed. 2015, 54, 14368-14371

[72] J. Gao, Y. Hori, T. Shimomura, M. Bordy, J. Hasserodt, K. Kikuchi, ChemBioChem 2020, 21, 656-662.

[73] E. Braselmann, A. J. Wierzba, J. T. Polaski, M. Chromiński, Z. E. Holmes, S.-T. Hung, D. Batan, J. R. Wheeler, R. Parker, R. Jimenez, et al., Nat. Chem. Biol. 2018, 14, 964-971.

[74] F. Bouhedda, K. T. Fam, M. Collot, A. Autour, S. Marzi, A. Klymchenko, M. Ryckelynck, Nat. Chem. Biol. 2020, 16, 69-76.

[75] J. Holleran, D. Brown, M. H. Fuhrman, S. A. Adler, G. W. Fisher, J. W. Jarvik, Cytometry A 2010, 77A, 776-782.

[76] Q. Yan, B. F. Schmidt, L. A. Perkins, M. Naganbabu, S. Saurabh, S. K. Andreko, M. P. Bruchez, Org. Biomol. Chem. 2015, 13, 2078-2086.

[77] Y. Wang, B. Ballou, B. F. Schmidt, S. Andreko, C. M. St Croix, S. C. Watkins, M. P. Bruchez, Chem. Commun. Camb. Engl. 2017, 53, 20012004

[78] C. Li, A. Mourton, M.-A. Plamont, V. Rodrigues, I. Aujard, M. Volovitch, T. Le Saux, F. Perez, S. Vriz, L. Jullien, et al., Bioconjug. Chem. 2018, 29, 1823-1828.

[79] K. F. Stensrud, K. J. Zanotti, A. S. Waggoner, B. A. Armitage, Photochem Photobiol. 2019, 95, 406-410. 
[80] C. Li, M.-A. Plamont, H. L. Sladitschek, V. Rodrigues, I. Aujard, P. Neveu, T. Le Saux, L. Jullien, A. Gautier, Chem. Sci. 2017, 8, 5598-5605.

[81] N. G. Bozhanova, M. S. Baranov, N. S. Baleeva, A. S. Gavrikov, A. S. Mishin, Int. J. Mol. Sci. 2018, 19, 3778.

[82] W. Song, R. L. Strack, N. Svensen, S. R. Jaffrey, J. Am. Chem. Soc 2014, 136, 1198-1201.

[83] Y. I. Meteleshko, A. V. Nemukhin, M. G. Khrenova, Photochem Photobiol. Sci. Off. J. Eur. Photochem. Assoc. Eur. Soc. Photobiol. 2019 18, 177-189.

[84] J. Torra, C. Lafaye, L. Signor, S. Aumonier, C. Flors, X. Shu, S. Nonell, G. Gotthard, A. Royant, Sci. Rep. 2019, 9, 2428.

[85] F. M. Pimenta, R. L. Jensen, T. Breitenbach, M. Etzerodt, P. R. Ogilby Photochem. Photobiol. 2013, 89, 1116-1126.

[86] R. Ruiz-González, A. L. Cortajarena, S. H. Mejias, M. Agut, S. Nonell, C Flors, J. Am. Chem. Soc. 2013, 135, 9564-9567.

[87] X. Shu, V. Lev-Ram, T. J. Deerinck, Y. Qi, E. B. Ramko, M. W. Davidson Y. Jin, M. H. Ellisman, R. Y. Tsien, PLoS Biol. 2011, 9, e1001041.

[88] M. Westberg, L. Holmegaard, F. M. Pimenta, M. Etzerodt, P. R. Ogilby, J. Am. Chem. Soc. 2015, 137, 1632-1642.

[89] M. Westberg, M. Bregnhøj, M. Etzerodt, P. R. Ogilby, J. Phys. Chem. B 2017, 121, 9366-9371.

[90] K. Makhijani, T.-L. To, R. Ruiz-González, C. Lafaye, A. Royant, X. Shu, Cell Chem. Biol. 2017, 24, 110-119.

[91] J. He, Y. Wang, M. A. Missinato, E. Onuoha, L. A. Perkins, S. C. Watkins, C. M. St. Croix, M. Tsang, M. P. Bruchez, Nat. Methods 2016, 13, 263268.

[92] J. Potzkei, M. Kunze, T. Drepper, T. Gensch, K.-E. Jaeger, J. Büchs, BMC Biol. 2012, 10, 28.

[93] R. Erapaneedi, V. V. Belousov, M. Schäfers, F. Kiefer, EMBO J. 2016 35, 102-113.

[94] H. Hu, A. Wang, L. Huang, Y. Zou, Y. Gu, X. Chen, Y. Zhao, Y. Yang, Free Radic. Biol. Med. 2018, 120, 255-265.

[95] A. Grover, B. F. Schmidt, R. D. Salter, S. C. Watkins, A. S. Waggoner, M. P. Bruchez, Angew. Chem. Int. Ed Engl. 2012, 51, 4838-4842.

[96] L. A. Perkins, Q. Yan, B. F. Schmidt, D. Kolodieznyi, S. Saurabh, M. B. Larsen, S. C. Watkins, L. Kremer, M. P. Bruchez, Biochemistry 2018, 57 861-871.

[97] T. Berbasova, S. Tahmasebi Nick, M. Nosrati, Z. Nossoni, E. M. Santos, C. Vasileiou, J. H. Geiger, B. Borhan, ChemBioChem 2018, 19, 12881295.

[98] Y. Shitashima, T. Shimozawa, T. Asahi, A. Miyawaki, Biochem. Biophys. Res. Commun. 2018, 496, 872-879.

[99] A. G. Tebo, F. M. Pimenta, M. Zoumpoulaki, C. Kikuti, H. Sirkia, M.-A Plamont, A. Houdusse, A. Gautier, ACS Chem. Biol. 2018, 13, 2392 2397.

[100] T.-L. To, B. J. Piggott, K. Makhijani, D. Yu, Y. N. Jan, X. Shu, Proc. Natl Acad. Sci. 2015, 112, 3338-3343.

[101] A. G. Tebo, A. Gautier, Nat. Commun. 2019, 10, 2822.

[102] T.-L. To, Q. Zhang, X. Shu, Protein Sci. Publ. Protein Soc. 2016, 25, 748-753.

[103] G. S. Filonov, V. V. Verkhusha, Chem. Biol. 2013, 20, 1078-1086.

[104] D. M. Shcherbakova, M. Baloban, A. V. Emelyanov, M. Brenowitz, P Guo, V. V. Verkhusha, Nat. Commun. 2016, 7, 12405.

[105] E. Tchekanda, D. Sivanesan, S. W. Michnick, Nat. Methods 2014, 11 641-644.

[106] D. Boassa, S. P. Lemieux, V. Lev-Ram, J. Hu, Q. Xiong, S. Phan, M Mackey, R. Ramachandra, R. E. Peace, S. R. Adams, et al., Cell Chem. Biol. 2019, 26, 1407-1416.e5 


\section{Table of Contents}

Chemogenetic optical reporters and actuators

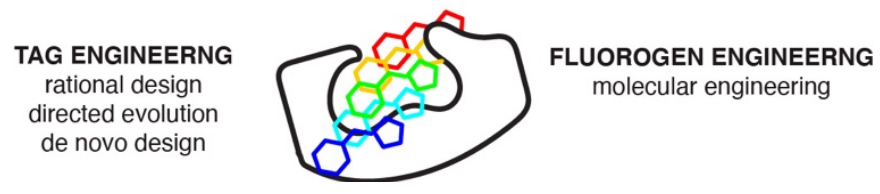

Chemists and biologists have recently built and breed a large collection of chemogenetic optical reporters and actuators enabling to visualize and study cellular biochemistry in new ways. 